\title{
MicroRNA expression profiling of specific cells in complex archival tissue stained by immunohistochemistry
}

\author{
Claudia Schuster ${ }^{1}$, Jan Budczies ${ }^{2}$, Claudius Faber ${ }^{1}$, Thomas Kirchner ${ }^{1}$ and Falk Hlubek ${ }^{1}$
}

Global implementation of molecular diagnostics in modern pathology has been limited by the use of formalin-fixed, paraffin-embedded (FFPE) tissues in current routine diagnostic procedures because of modification and degradation of nucleic acids and protein molecules. In particular, molecular analysis of a specific cell type potentially important for biomarker identification is largely prevented in highly complex, solid tissues routinely used in histopathology. Accumulating data report on the substantial contribution of microRNA molecules (miRNA) to tumor development and malignant progression of most human malignancies. Our objective was to establish a sensitive and robust procedure to quantify miRNA expression in specific cells from complex archival tumor tissues identified by immunohistochemistry. Here, we show reliable detection of miRNA expression profiles determined from limited amounts of colorectal cancer FFPE tissues after routine staining procedure. The combination of routinely used FFPE specimens stained by immunohistochemistry with the molecular analysis of lasermicrodissected complex tumor tissue resulted in robust miRNA expression patterns exclusively obtained from epithelial tumor cells. This approach allows for a detailed molecular analysis of cancer cells and distinct stromal cell types and their in situ interaction in solid tumors. Hence, the methodology can offer new perspectives for basic research and, by comprehensive use of present archival tissue collections linked to clinical databases, facilitate miRNA biomarker identification in defined tissue cells for future diagnostic and therapeutic strategies.

Laboratory Investigation (2011) 91, 157-165; doi:10.1038/labinvest.2010.134; published online 26 July 2010

KEYWORDS: archival tissue; colorectal cancer; expression profiling; FFPE; immunohistochemistry; microRNA

Current routine diagnostics in pathology mainly depend on the assessment of tissue cell morphology and the detection of protein marker expression by immunohistochemistry. For decades, formalin fixation followed by embedding in paraffin (formalin-fixed, paraffin-embedded, FFPE) has been used for tissue fixation in routine pathological diagnostics, particularly to preserve tissue morphology. As knowledge of the molecular processes of malignant diseases has made an immense progress, the realization of a personalized medicine avoiding therapeutic side effects and optimizing treatment efficiency has become feasible, and molecular tools for diagnostic purposes have become important. Moreover, as solid tumors are highly complex tissues, a combination of morphology assessment by classical immunohistochemical staining and molecular analysis is highly desirable in order to enable biomarker identification in specific, potentially relevant cells for molecular diagnostics and individual therapy.

However, compatibility of current routine diagnostics with molecular analyses is very limited, as tissue fixation of FFPE material and immunohistochemical procedures are a major source of high nucleic acid degradation and modification. Formalin fixation is known to cross-link tissue proteins with each other and with DNA or RNA molecules, to preserve cellular structures. Consequently, formalin fixation traps nucleic acids and prevents their extraction for molecular analyses. In addition, formalin modifies RNA by adding mono-methylol groups to all the four bases blocking subsequent molecular applications, such as reverse transcription. ${ }^{1}$ RNA fragmentation may also result from time to fixation (tissue autolysis) and duration of fixation, as well as

\footnotetext{
${ }^{1}$ Institute of Pathology, Ludwig-Maximilians-University of München, München, Germany and ${ }^{2}$ Institute of Pathology, Charité-Universitätsmedizin, Berlin, Germany Correspondence: Dr F Hlubek, Institute of Pathology, Ludwig-Maximilians-University of München, Thalkirchner Straße 36, München 80337, Germany. E-mail: falk.hlubek@med.uni-muenchen.de 
elevated temperature conditions during tissue embedding and immunohistochemical staining procedures.

Recently, naturally occurring small, non-coding RNAs (miRNAs), which negatively regulate gene expression mainly at the level of protein synthesis, have been described. The number of miRNAs identified is constantly increasing, and it has become clear that miRNAs are one of the largest systems of gene regulation. MiRNAs are likely to be important for future diagnostics, as they represent an evolutionary highly conserved system and control crucial cellular processes such as proliferation, differentiation, and apoptosis. ${ }^{2,3}$ There are numerous studies demonstrating that miRNAs have important roles in different human malignancies, ${ }^{4-6}$ which is supported by the fact that the expression of many miRNAs is deregulated in human tumors by gene amplification or deletion. ${ }^{7-9}$ Furthermore, an increasing number of reports indicate that miRNAs can have oncogenic as well as tumorsuppressive functions (for review see Visone and Croce, ${ }^{6}$ Hammond, ${ }^{10}$ and Croce ${ }^{11}$ ).

In contrast to mRNA expression profiling, miRNA signatures have proven to successfully distinguish tumors according to their grade of differentiation. ${ }^{2,9}$ Proof of principle that miRNAs are suitable tools for diagnostics was provided by $\mathrm{Lu}$ and colleagues, who were able to categorize undifferentiated tumors depending on the miRNA expression signatures according to their specific tissue origin. ${ }^{2,9}$ These tumors could not be differentiated by classical histology. Moreover, Takamizawa et $a l^{12}$ demonstrated that reduced expression of specific let-7 homologous miRNAs in human lung tumors significantly correlates with poor prognosis of disease development, suggesting the use of let- 7 for diagnostic purposes. ${ }^{2}$

In general, miRNAs are highly suitable for diagnostic applications, as several studies have shown that miRNA molecules can be isolated without difficulty from FFPE tissue routinely used in diagnostics. ${ }^{13}$ This is of importance, for large archives of FFPE tissue samples exist in pathology departments worldwide, being a highly valuable source of well-characterized tissues from diverse malignancies for retrospective studies. In addition, clinical patient data on disease progression is available for many of these tissue samples in tumor repositories or clinics. Hence, identification and application of molecular biomarkers for tumor aggressiveness and therapy resistance in routine diagnostics may become feasible based on miRNA expression signatures to realize an individualized medicine approach.

The principle objective of this study was to combine classical diagnostics based on immunohistochemistry and current molecular analysis to reveal the molecular characteristics of specific cells in complex tumor tissues. We investigated whether miRNA recovery and expression profiling is contrivable from limited numbers of cells in routinely fixed tissue defined by immunohistochemical staining procedures.

\section{MATERIALS AND METHODS}

\section{Tissue Specimen and Immunohistochemistry}

FFPE colorectal adenocarcinomas from patients who underwent surgery without additional treatments were retrieved from the archive of the Institute of Pathology, University of Munich (LMU). Serial sections of FFPE tissue samples, archived between 1995 and 2005, were mounted on membrane slides (MMI/Olympus, Hamburg, Germany) and deparaffinized in xylene by incubation at room temperature for $20 \mathrm{~min}$, substituting xylene after $10 \mathrm{~min}$. Following two washing steps with $100 \%$ ethanol, samples were air dried and stained for $\beta$-catenin. Immunohistochemistry for $\beta$-catenin was performed using an anti- $\beta$-catenin antibody (Epitomics/ Biomol 1247-1, 1:150; Hamburg, Germany) as described previously $^{14}$ with the following modifications: antigen retrieval was performed using $1 \times$ TUF buffer (PanPath, Budel, The Netherlands) at $90^{\circ} \mathrm{C}$ or $98^{\circ} \mathrm{C}$, respectively. Incubation times were according to Vectastain Universal Quick Kit manual (Linaris, Wertheim, Germany). Tumor cells positive for $\beta$-catenin were subsequently isolated by laser microdissection using SL $\mu$ Cut (MMI/Olympus), avoiding contamination with stromal cells.

Alternatively, deparaffinized sections were stained with Mayer's hemalaun (Merck, Darmstadt, Germany) for $5 \mathrm{~s}$, followed by $3 \mathrm{~s}$ in DEPC-treated water and $1 \mathrm{~min}$ in $70 \%$ ethanol. Subsequently, samples were air-dried and tumor morphology was confirmed by a pathologist.

\section{RNA Isolation from FFPE Samples}

After laser microdissection, total RNA was extracted from isolated cells using PKD buffer and proteinase $\mathrm{K}$ digestion at $55^{\circ} \mathrm{C}$ (Qiagen, Hilden, Germany) for $5 \mathrm{~h}$, followed by RNA precipitation with $200 \mathrm{mM}$ sodium acetate and isopropanol at $-80^{\circ} \mathrm{C}$. After washing, total RNA, containing the miRNA fraction, was resuspended in nuclease-free water. RNA integrity was assessed by microcapillary electrophoresis (Experion, BioRad, München, Germany).

\section{Reverse Transcription}

Reverse transcription reactions for individual and multiplex assays were performed using the TaqMan Reverse Transcription Kit (Applied Biosystems, Weiterstadt, Germany) according to the manufacturer's instructions. For single RT reactions, miRNA-specific RT primer were used, whereas for multiplex-RT assays, multiplex-RT pools (Applied Biosystems) were used. Up to 48 miRNAs were converted into cDNA in one reaction. The reaction conditions were as follows: 60 cycles of $30 \mathrm{~s}$ at $20^{\circ} \mathrm{C}, 30 \mathrm{~s}$ at $42^{\circ} \mathrm{C}, 1 \mathrm{~s}$ at $50^{\circ} \mathrm{C}$, followed by $5 \mathrm{~min}$ at $85^{\circ} \mathrm{C}$ for enzyme inactivation.

\section{Pre-PCR and Real-Time Quantification of microRNAs}

Subsequently, the RT products were amplified using sequence-specific primers according to the protocol of Tang et $a l^{15}$ in order to generate sufficient amounts of template. Briefly, reaction mixes contained $0.05 \times$ multiplex primer 
pool (Applied Biosystems), 6.25 U AmpliTaq Gold, $2 \mathrm{mM}$ $\mathrm{MgCl}_{2}, 2 \mathrm{mM}$ dNTP, $1 \times$ TaqMan Universal Master Mix (Applied Biosystems). The reactions were incubated at $95^{\circ} \mathrm{C}$ for $10 \mathrm{~min}$, followed by $16 \mathrm{cycles}$ of $95^{\circ} \mathrm{C}$ for $1 \mathrm{~s}$ and $65^{\circ} \mathrm{C}$ for $1 \mathrm{~min}$.

Preamplified PCR products were analyzed by qRT-PCR using TaqMan miRNA assays (Applied Biosystems) according to the instructions of the manufacturer. TaqMan miRNA assays are designed to detect and quantitate mature miRNAs with high sensitivity and specificity. The PCR reaction mixes contained $1 \times$ TaqMan Universal Master Mix, $20 \times$ TaqMan assay and RT products and were analyzed on a LightCycler 480 instrument (Roche Diagnostics, Mannheim, Germany). The temperature profile consisted of: $10 \mathrm{~min}$ at $95^{\circ} \mathrm{C}$, followed by 40 cycles of $95^{\circ} \mathrm{C}$ for $15 \mathrm{~s}$, and $60^{\circ} \mathrm{C}$ for $1 \mathrm{~min}$. Single-plex real-time PCRs were run in triplicates, whereas multiplex analyses were duplicated.

\section{qRT-PCR Data Processing}

The assessment of miRNA expression was determined by the $\Delta \Delta C_{\mathrm{t}}$ comparative threshold method $\left(\Delta \Delta C_{\mathrm{t}}\right) \cdot{ }^{16}$ In this study, we used TaqMan MicroRNA assays for real-time quantification of microRNAs according to the manufacturer's instructions, recommending SNORD48 (alias RNU48) for normalization in tissue specimens (Applied Biosystems, Application Note TaqMan MicroRNA assays 2007). SNORD48 was determined as the most stably expressed control gene and all miRNA expression data were normalized using SNORD48.

\section{Statistical Analysis}

miRNA expression patterns were analyzed using one-way analysis of variance (ANOVA). The significance for a consistent variation of the normalized expression levels of six miRNAs over nine patients was assessed by applying the F-test. For post hoc analysis, Tukey's honest significant difference method was used and significance of differential expression between two miRNAs was assessed by Tukey adjusted $P$-values. ${ }^{17,18} P$-values $<0.05$ were considered as significant.

\section{RESULTS \\ MicroRNA Expression Analysis in Small Samples of Archival FFPE Tissue}

In order to investigate the sensitivity of miRNA quantification in limited amounts of FFPE material, we used real-time RT-PCR analysis to determine the expression level of miR16 , a ubiquitiously expressed and medium abundant miRNA. For this purpose, we isolated $\sim 100$ cells by lasermicrodissection from human archival FFPE colorectal tumor tissue. The analysis indicated that miR-16 expression was detectable with a $C p$-value of $\sim 35$. For reliable detection of less abundantly expressed microRNAs, however, 16 cycles of PCR preamplification were included in the procedure after RNA reverse transcription in order to generate sufficient amounts of template cDNA for subsequent quantitative
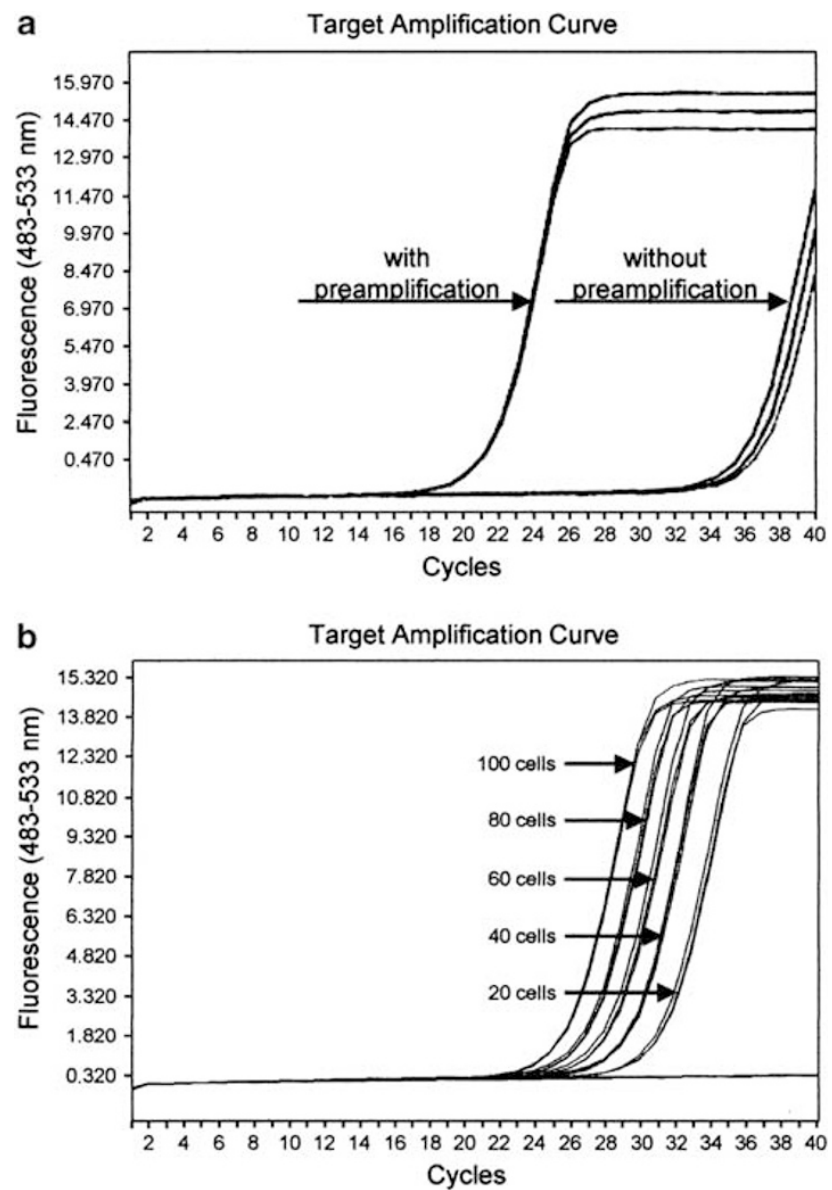

Figure 1 Detection of miR-16 expression in small samples of archival FFPE tissue. Approximately 100 tumor cells were isolated by lasermicrodissection from hemalaun-stained colorectal carcinoma tissue and miR-16 expression was determined by quantitative real-time PCR in triplicates with or without 16 cycles of previous CDNA amplification. Preamplification substantially increased miR-16 detection sensitivity reducing the mean $C p$ from $\sim 35-$ 20.8. The increasing fluorescence intensity in correlation to the number of PCR cycles is shown (a). Effect of decreasing cell numbers $(100,80,60,40$, and 20 cells) isolated from FFPE tissue on miR-16 detection sensitivity. Reliable detection of miR-16 expression was obtained in as few as 20 cells of archival FFPE tissue (b). No template controls relate to the baseline.

real-time PCR. By including preamplification, the sensitivity of miR-16 detection was increased by about 4.5 log ranges (Figure 1a). Having established this protocol, we assessed the dynamic range and sensitivity of the miR-16 quantification by reducing cell numbers obtained by lasermicrodissection. Figure $1 \mathrm{~b}$ illustrates the effect of serial reduction of cell numbers on detection of miR-16 levels. One hundred, 80, 60, 40 , and 20 cells were isolated and miR-16 expression was quantified. The results indicate that miR-16 is reliably detectable in as few as 20 cells retrieved from archival FFPE tissue.

Effect of Immunohistochemical Staining on RNA Quality To determine the impact of tissue staining procedures on the quality of RNA isolated from FFPE tissue, serial sections from 
the same colorectal carcinoma FFPE-tissue sample were prepared and subjected to different staining protocols. As tissue exposure to high temperature during staining procedures is presumed to be a critical factor for RNA quality, we compared the following three staining procedures: $\beta$-catenin-specific immunohistochemistry at $98^{\circ} \mathrm{C}$ (regular temperature for antigen retrieval), $\beta$-catenin-specific immunohistochemistry at $90^{\circ} \mathrm{C}$ (reduced temperature for antigen retrieval), and hemalaun staining as control experiment. In contrast to $\beta$-catenin immunohistochemical staining, the hemalaun staining is a histochemical staining procedure without heating step, frequently used for characterization of tissue morphology.

The antigen retrieval temperature during immunohistochemical $\beta$-catenin staining had only minor influence on the staining quality with slightly less intense nuclear staining at $90^{\circ} \mathrm{C}$ (Figure 2a). However, at a lower temperature for antigen retrieval, the staining was not successful $\left(80^{\circ} \mathrm{C}\right.$, data not shown). The RNA quality of all samples was assessed by capillary electrophoresis. Figure $2 \mathrm{~b}$ illustrates, that in comparison with intact total RNA from colorectal culture cells (\#4), 18 S ribosomal RNA and 28 S ribosomal RNA peaks were detectable neither in hemalaun-stained FFPE tissue samples (\#1) nor in samples stained by immunohistochemistry $(\# 2,3)$, indicating a high degree of RNA degradation. Therefore, we conclude that the application of different temperature profiles during the staining process had only a minor impact on total RNA quality determined by capillary electrophoresis. However, compared with hemalaun-stained tissue, $\beta$-catenin-stained samples exhibited a less prominent peak of RNA at the size of $\sim 130$ nucleotides, representing the majority of RNA molecules isolated from FFPE material. Although total RNA quality was poor in FFPE tissues, we analyzed miRNA expression levels in samples subjected to these varying temperature profiles during $\beta$-catenin staining. To evaluate the effect of the staining conditions on miRNA profiling, we extended the analysis to miRNAs with different expression levels. Five miRNAs were selected and relative expression levels, using RNU48 as endogenous calibrator, were determined in hemalaun and $\beta$-catenin-stained tissue samples. Figure 3 shows relative expression levels of individual miRNAs in tumor tissue obtained from serial sections of colorectal FFPE-tissue samples. The resulting miRNA expression patterns of the replicates using different temperature profiles during immunohistochemistry showed high correlation with miRNA expression in hemalaun-stained samples, which were not exposed to high temperatures during the staining procedure (Figure 3). Hence, these results corroborate that miRNA expression can be reliably quantified in archival FFPE tissue stained by immunohistochemistry.

\section{Multiplex microRNA Expression Analyses in Laser Microdissected Archival FFPE Material}

Although the method presented is clearly suitable for detection of single miRNAs in tumor tissue obtained from IHCstained archival FFPE samples, we next determined whether comprehensive miRNA profiles could be reliably generated from small cell numbers of specific tumor cells. We isolated $\sim 200$ tumor cells using lasermicrodissection and analyzed a pool of 34 miRNAs expected to be expressed at different levels. The miRNA quantification was highly sensitive, discriminating miRNA expression over a dynamic range of $5 \mathrm{log}$ ranges (Figure 4). For clinical diagnostics, not only sensitivity and specificity, but also reproducibility and robustness are essential parameters for reliable miRNA profiling. In addition, miRNA expression analysis of specific cells in a complex tissue identified by immunohistochemistry is highly desirable. For this purpose, only $\beta$-catenin-expressing tumor cells, identified by immunohistochemistry, were isolated by lasermicrodissection (Figure 5a). The reliability of miRNA expression profiling in such small cell numbers was tested using independently generated cDNAs from the same tissue samples (technical replicates). In Figure 5b, normalized $C p$ values of significantly detected miRNAs of the individual expression analyses are shown as correlation scatter plots with the initial experimental data on the $x$ axis and the replication data on the $y$ axis. miRNA profiles from technical replicates proved to be highly correlated with a coefficient of $r^{2}=0.982$ and $r^{2}=0.969$, respectively. In contrast, miRNA profiles from different patients showed, as expected, less significant correlation $\left(r^{2}=0.548\right.$; Figure $\left.5 c\right)$. These data indicate that multiplex miRNA expression analysis of FFPE tissue stained by routine immunohistochemistry using quantitative real-time PCR is highly reproducible.

To further corroborate the robustness of miRNA expression profiling of specific cells identified by immunohistochemistry, $\beta$-catenin-expressing tumor cells were isolated by lasermicrodissection from well to moderately differentiated, microsatellite stable colorectal carcinoma tissues of nine different patients. Multiplex reverse transcription and preamplification were performed for each sample independently and individual quantification of six arbitrarily chosen miRNAs of different expression levels was determined by real-time PCR. On the basis of these results, we plotted the normalized expression data from nine biological replicates for each miRNA, and by ANOVA analysis we observed significant differences in the expression levels among the six miRNAs (Figure 6; $P=2.2 \times 10^{-24}$ ). Further analysis using Tukey's test showed statistical significance of differential expression between 13 miRNA pairs out of 15 possible combinations. These experiments demonstrate that this method enables robust detection of miRNA expression patterns in tumor samples (see Supplementary Figure 1 for more expression data). Together, the results strongly suggest that miRNA expression profiling in routinely used archival FFPE tissue after immunohistochemical staining is a robust method, allowing for sensitive analyses of small amounts of specific cells in complex tissues.

\section{DISCUSSION}

Over the last decade, mRNA expression profiling has been studied extensively and molecular diagnostic tools have 
a

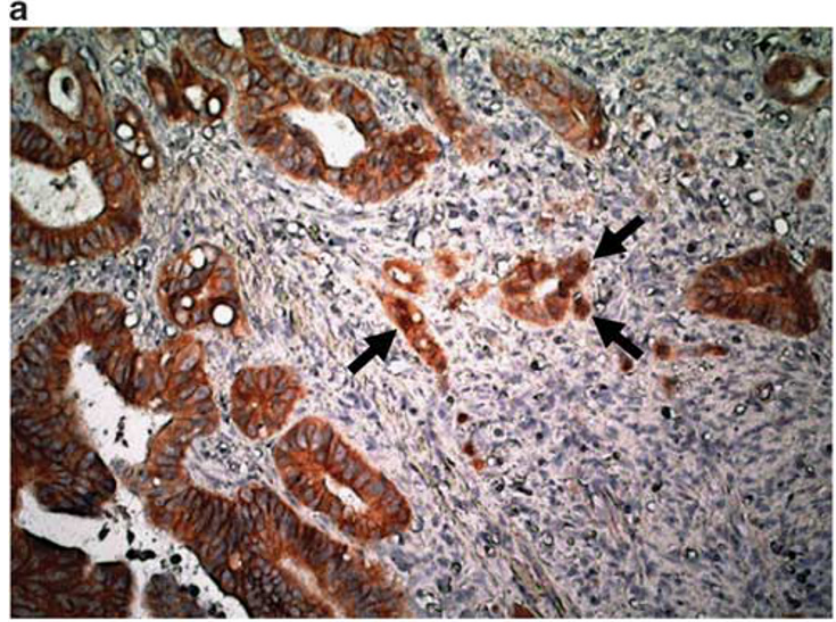

b

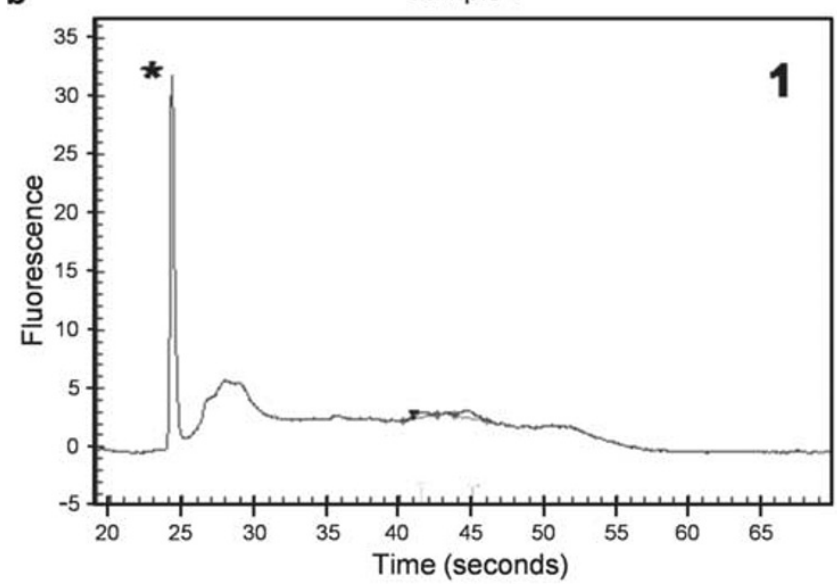

Sample 3

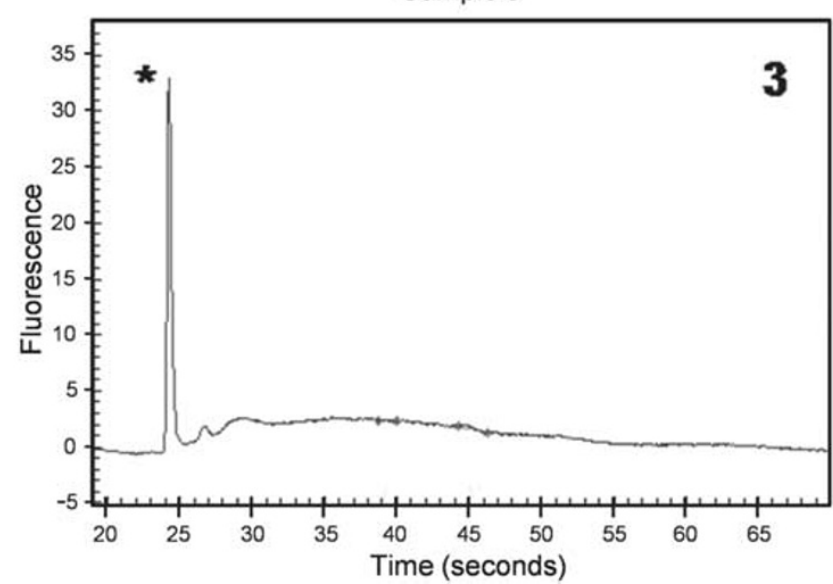

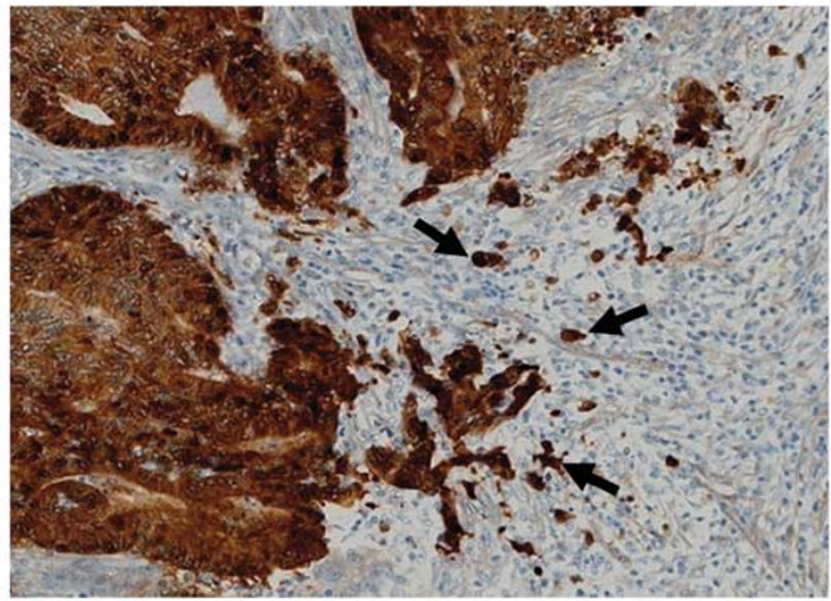

Sample 2

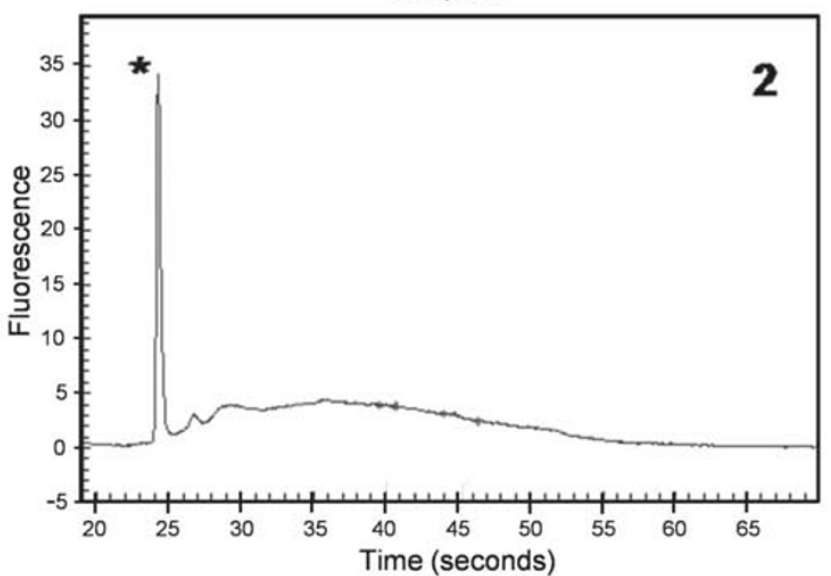

control RNA

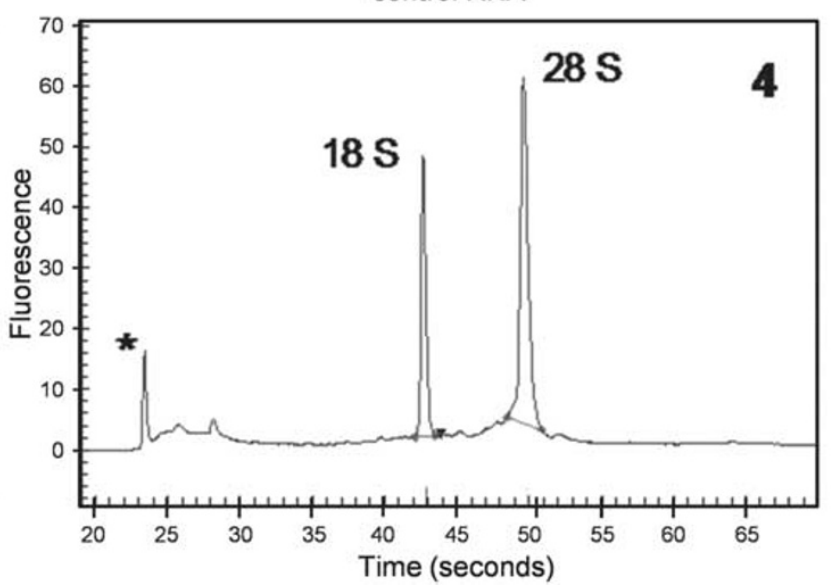

Figure 2 (a) Minor difference in quality of immunohistochemical staining after antigen retrieval at $90^{\circ} \mathrm{C}$ (left panel) or $98^{\circ} \mathrm{C}$ (right panel). At lower temperature conditions, only slightly reduced nuclear staining of $\beta$-catenin was observed. Black arrows indicate tumor cells expressing nuclear $\beta$-catenin at the invasive front. (b) The impact of immunohistochemical staining procedures on RNA integrity. Individual slides of serial sections of the same FFPE-tissue sample were stained by different procedures and total RNA was extracted. RNA quality from each section was analyzed by capillary electrophoresis.

The electropherograms corresponding to the staining procedures are shown. (1) Hemalaun staining, (2) $\beta$-catenin-specific immunohistochemistry at $98^{\circ} \mathrm{C}$, (3) $\beta$-catenin-specific immunohistochemistry at $90^{\circ} \mathrm{C}$, (4) unstained cultured cells. The asterisk (*) marks the lower marker peak of 50 bases at the far left of the electropherogram. 


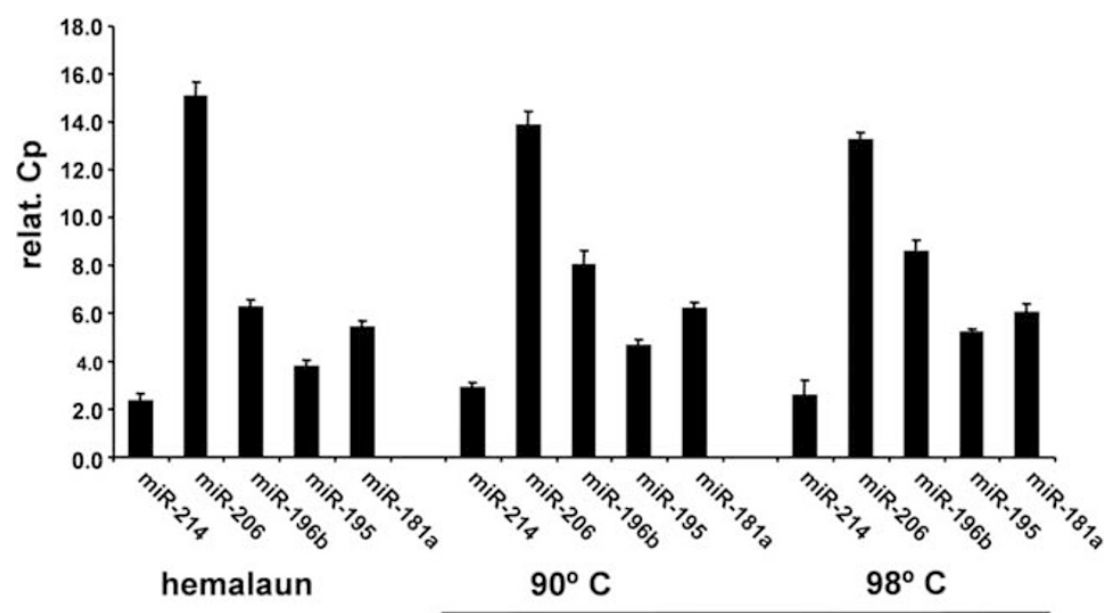

immunohistochemistry

Figure 3 Correlation of miRNA expression patterns in tissues treated with different staining procedures. Relative expression of five miRNAs was quantified by real-time PCR in serial sections of the same FFPE-tissue sample following different immunohistochemical staining procedures with high temperature antigen retrieval at 90 and $98^{\circ} \mathrm{C}$. Note, the miRNA expression pattern after immunohistochemical staining strongly resembles the miRNA expression in hemalaun-treated samples, a staining without high temperature treatment. miRNAs were measured in triplicates and normalized to the expression of RNU48 control RNA $(\Delta C p)$. Standard deviations are indicated.

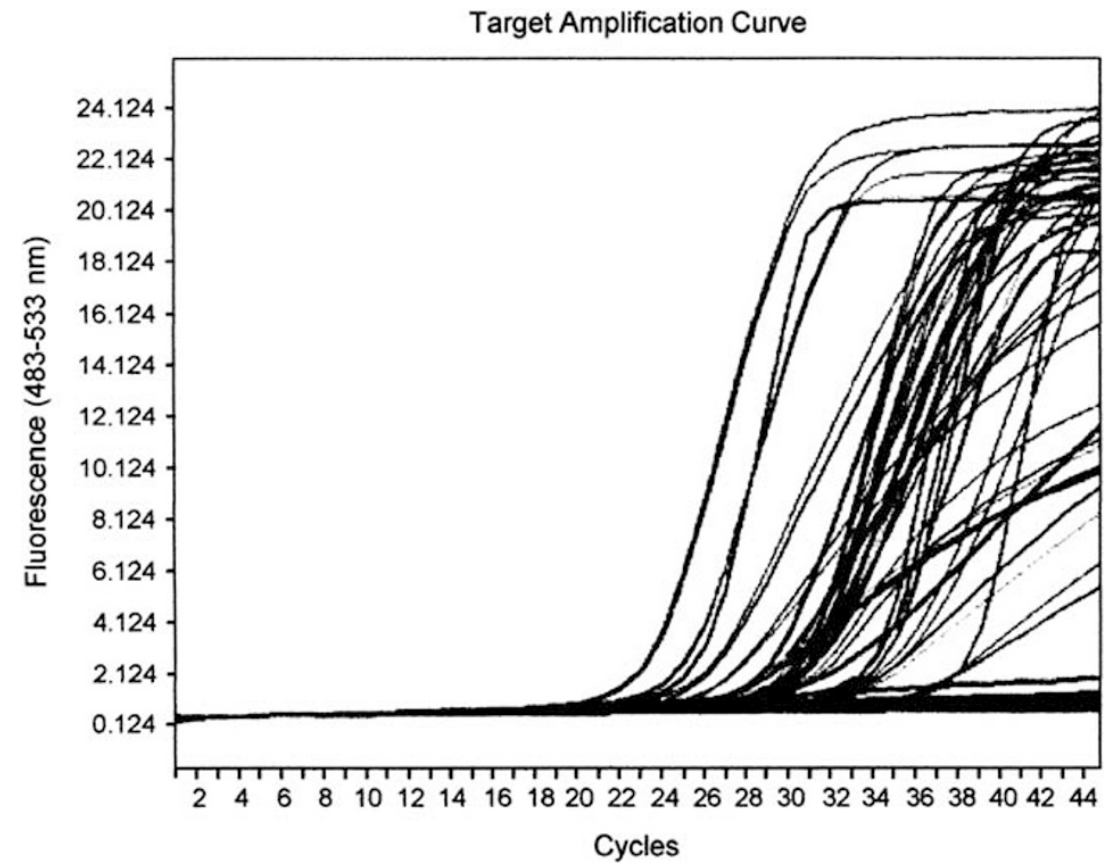

Figure 4 Detection range of miRNA expression profiles generated from small numbers of tumor cells. Two hundred tumor cells were isolated by lasermicrodissection from hemalaun-stained colorectal cancer tissue. After 16 cycles of multiplexed preamplification, we analyzed a pool of 34 miRNAs expressed at different levels by discriminating miRNA expression over $\sim 5$ log ranges. The fluorescence intensities in correlation to the number of real-time PCR cycles are shown.

been developed, but so far only very few tests have been implemented in clinical practice. ${ }^{19,20}$ Recent reports suggest that miRNA expression profiles have the potential of clinical biomarkers for diagnosis and prognosis and some miRNAs may serve as promising targets for novel therapies (for review see Visone and Croce, ${ }^{6}$ Aslam et al, ${ }^{21}$ Negrini et al, ${ }^{22}$ Wang et $a l,{ }^{23}$ Paranjape et $a l^{24}$ and Garzon et $\left.a l^{25}\right)$. Compared with mRNA, miRNA has the advantage to be considerably more 

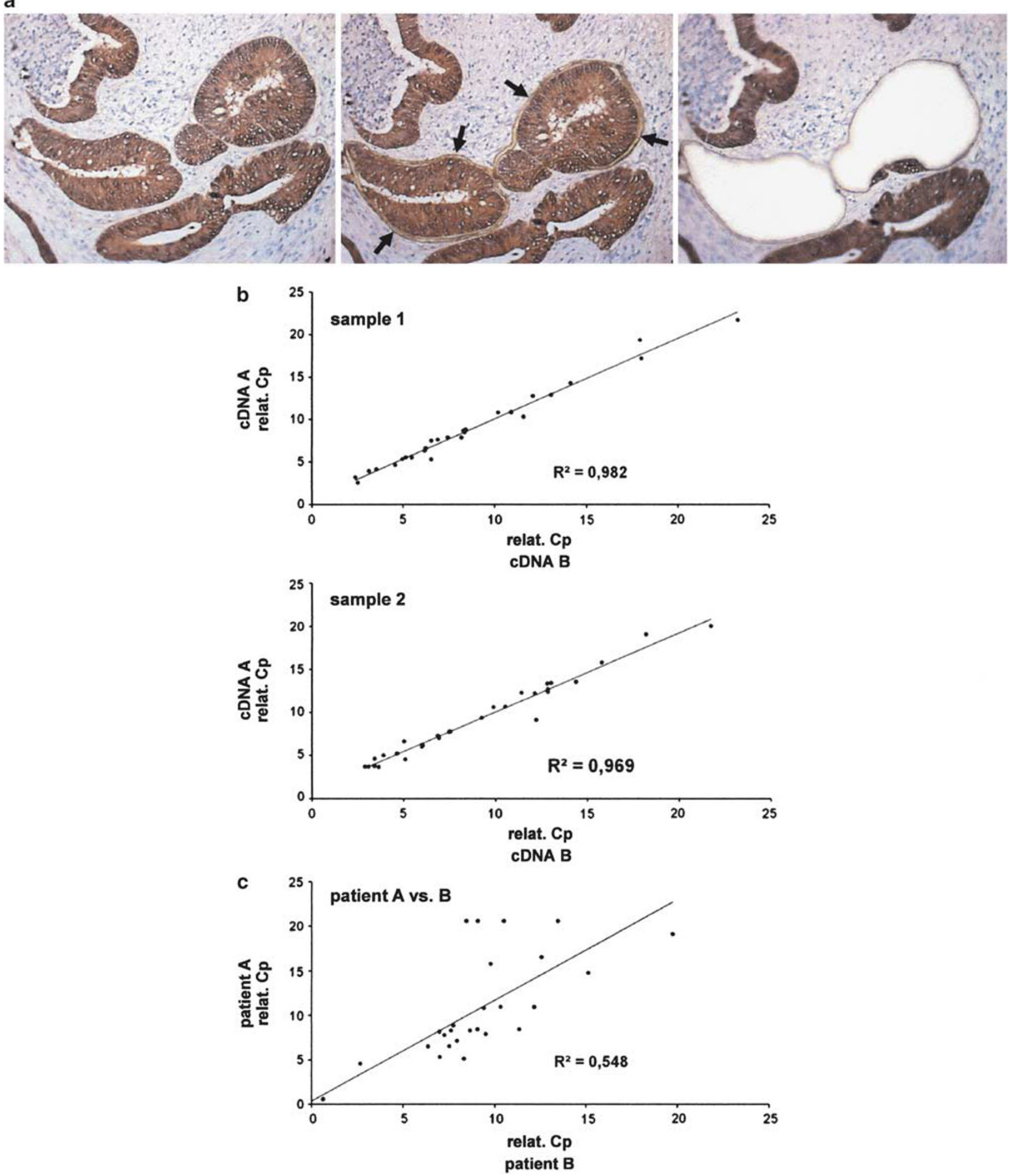

Figure 5 (a) Reliability of miRNA profiling from $\sim 200$ tumor cells in FFPE tissue. $\beta$-catenin-expressing tumor cells identified by immunohistochemistry were isolated by lasermicrodissection from colorectal carcinoma tissue. (Left) $\beta$-catenin-stained tissue; (middle) tissue cut by laser (arrows); (right) tissue after specific recovery of $\beta$-catenin-positive tumor cells $(5 \mu \mathrm{m}$ section). (b) High reproducibility of miRNA expression profiling in small numbers of tumor cells (ca. 200 cells) by multiplexed quantitative PCR in FFPE tissue, stained by immunohistochemistry. For technical replicates, independent CDNA synthesis, amplification and qPCR analysis was performed twice for two different tumor samples (sample 1 and sample 2). The correlation scatter plots show normalized $C p$ values $(\Delta C p)$ of detected miRNAs of the individual expression analyses with the initial experimental data on the $x$ axis and the replication data on the $y$ axis. Correlation coefficients of $r^{2}=0.982$ and $r^{2}=0.969$, respectively, indicate high correlation of miRNA profiles from different cDNA preparations of the same tissue samples. (c) By contrast, miRNA profiles from different patients showed, as expected, less significant correlation $\left(r^{2}=0.548\right)$. 


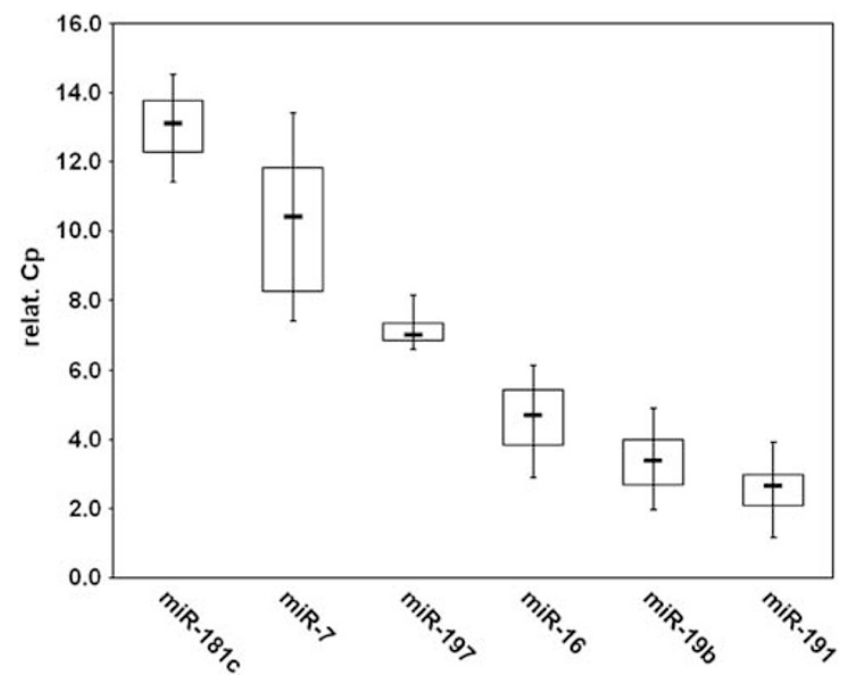

Figure 6 Robust miRNA expression profiling in limited cell numbers isolated by lasermicrodissection from FFPE-tumor tissue stained by immunohistochemistry. Colorectal carcinoma samples of nine patients were stained for $\beta$-catenin. Only $\beta$-catenin-expressing tumor cells were selected for isolation by lasermicrodissection. The expression of six selected miRNAs of different expression level was determined by real-time PCR subsequent to multiplexed preamplification. For each miRNA ( $x$ axis), the $C p$ values normalized to RNU48 $(\Delta C p)$ of nine patients were calculated. Gray bars represent the mean relative expression values. ANOVA analysis detected significant variations in miRNA expression levels of the six miRNAs consistent over the tissue samples of all patients $\left(P=2.2 \times 10^{-24}\right)$. Further analysis using Tukey's honest significance test revealed significant differential miRNA expression analyzed pairwise for all combinations with the exception of miR-19b vs miR-191 and miR-16 vs miR-19b. The box defines upper and lower quartiles ( $25 \%$ and $75 \%$, respectively) and the error bars indicate upper and lower adjacent limits.

stable and is easy to extract from archival FFPE tissues. ${ }^{26,27}$ MiRNA quality neither changes with storage time of FFPE tissue (Supplementary Figure 2) nor with formalin fixation time and does not, as reported here, correlate with the RNA quality assessed by capillary electrophoresis. ${ }^{27}$ Notwithstanding the negative impact of fixation and embedding of tissue samples on the quality of RNA, miRNA quantification by real-time PCR yielded robust and reproducible results. ${ }^{28}$ Comparison of miRNA expression patterns in FFPE $v s$ fresh frozen samples resulted in a high correlation between both types of samples, emphasizing the usefulness of FFPE material as a valuable source for miRNA detection. ${ }^{29,30}$ In contrast to our study, previous work mainly focused on array-based profiling technologies, which are requiring high amounts of input RNA. In these studies, whole sections or macrodissected regions were used as starting material. However, tumors are complex tissues containing numerous different cell types such as diverse stromal cells and tumor cells. In addition, cancerous tissues include different tumor regions, suggesting intratumorous heterogeneity. In our previous work, we could detect significant differential gene expression between distinct regions (eg invasion front, tumor center) of human colorectal tumors demonstrating heterogeneity on the molecular level. ${ }^{31}$

To investigate specific cell types of complex tissues, we combined immunostaining procedures allowing for unambiguous cell identification for lasermicrodissection with miRNA expression analysis to allow precise excision and molecular analysis of the cells of interest.

Here, we show successful quantification of miRNA expression in FFPE-tissue sections after immunohistochemical staining resulting in robust miRNA expression profiles indicated by good correlation between technical as well as biological replicates. Although the reason for the high stability of miRNA is unclear, it has been presumed that the small size and the secondary structure may contribute to the stability. ${ }^{27}$ The small molecule size might facilitate faster and complete release from the tissue during the isolation procedure, because of fewer cross-links that have been established during fixation. ${ }^{26}$ Further considerations suggest that miRNAs might be protected from damage by heat or other adverse conditions, because they are (unlike mRNA molecules) embedded in protein complexes, for example the RNA-induced silencing complex. The high stability of miRNAs is advantageous as it enables large retrospective studies directly using the archival tissues routinely stored in the pathology departments world wide with the corresponding clinical data available. The combination of classical immunohistochemistry and molecular analysis based on preamplification and miRNA expression analysis described here has the additional benefit to facilitate molecular diagnostics on very limited amounts of formalin-fixed tissues, such as biopsies. Most importantly, the technique presented permits molecular analysis of specific and potentially critical cells within highly complex tissues identified by immunohistochemical staining and precisely isolated by lasermicrodissection. This approach facilitates the independent, molecular analysis of diverse cell types, such as tumor cells and stromal cells, within a complex tissue to analyze their molecular phenotype and cellular interactions in the in vivo context.

Taken together, our results indicate that miRNAs are easy to extract from FFPE tissue, even after immunohistochemical treatment used in pathological diagnostics. MiRNAs are accordingly highly valuable as putative molecular markers, the detection and quantification of which can be seamlessly integrated in current diagnostic procedures without the need of collecting additional fresh frozen tissue or new tissue sectioning. On the basis of these results, fast, cost-effective and less labor-intensive procedures of molecular diagnostics could be established and integrated into routine diagnostic procedures in pathology without reorganization of the current laboratory process. More importantly, the data presented demonstrate the feasability of molecular analysis of a specific cell type identified by routine immunohistochemistry within highly complex, solid tumor tissues enabling new perspectives in biomarker identification and individual molecular diagnostics. 
Supplementary Information accompanies the paper on the Laboratory Investigation website (http://www.laboratoryinvestigation.org)

\section{ACKNOWLEDGEMENTS}

We thank A Sendelhofert, A Heier, and S Pfeiffer for expert technical assistance. This work was supported by the Wilhelm Sander-Stiftung (grant no. 2009.063.1) to $\mathrm{FH}$ and TK.

\section{DISCLOSURE/CONFLICT OF INTEREST}

The authors declare no conflict of interest.

1. Masuda N, Ohnishi $\mathrm{T}$, Kawamoto $\mathrm{S}$, et al. Analysis of chemical modification of RNA from formalin-fixed samples and optimization of molecular biology applications for such samples. Nucl Acids Res 1999;27:4436-4443.

2. Esquela-Kerscher A, Slack FJ. Oncomirs-microRNAs with a role in cancer. Nat Rev Cancer 2006;6:259-269.

3. Bartel DP. MicroRNAs: genomics, biogenesis, mechanism, and function. Cell 2004;116:281-297.

4. Couzin J. MicroRNAs make big impression in disease after disease. Science 2008:319:1782-1784.

5. Croce CM, Calin GA. miRNAs, cancer, and stem cell division. Cell 2005;122:6-7.

6. Visone R, Croce CM. MiRNAs and cancer. Am J Pathol 2009;174 1131-1138.

7. Michael MZ, O'Connor SM, van Holst Pellekaan NG, et al. Reduced accumulation of specific microRNAs in colorectal neoplasia. Mol Cancer Res 2003;1:882-891.

8. He L, Thomson JM, Hemann MT, et al. A microRNA polycistron as a potential human oncogene. Nature 2005;435:828-833.

9. Lu J, Getz G, Miska EA, et al. MicroRNA expression profiles classify human cancers. Nature 2005;435:834-838.

10. Hammond SM. MicroRNAs as oncogenes. Curr Opin Genet Dev 2006;16:4-9.

11. Croce CM. Causes and consequences of microRNA dysregulation in cancer. Nat Rev Genet 2009:10:704-714.

12. Takamizawa J, Konishi $\mathrm{H}$, Yanagisawa $\mathrm{K}$, et al. Reduced expression of the let-7 microRNAs in human lung cancers in association with shortened postoperative survival. Cancer Res 2004;64:3753-3756.

13. Siebolts $\mathrm{U}$, Varnholt $\mathrm{H}$, Drebber $\mathrm{U}$, et al. Tissues from routine pathology archives are suitable for microRNA analyses by quantitative PCR. J Clin Pathol 2009;62:84-88.

14. Hlubek $F$, Spaderna $S$, Jung $A$, et al. beta-Catenin activates a coordinated expression of the proinvasive factors laminin-5 gamma2 chain and MT1-MMP in colorectal carcinomas. Int J Cancer 2004; 108:321-326.
15. Tang $F$, Hajkova $P$, Barton SC, et al. MicroRNA expression profiling of single whole embryonic stem cells. Nucleic Acids Res 2006;34:e9.

16. Livak KJ, Schmittgen TD. Analysis of relative gene expression data using real-time quantitative PCR and the 2(-Delta Delta $C(T)$ ) method. Methods 2001;25:402-408.

17. Hsu JC. Multiple Comparisons: Theory and Methods. Chapman \& Hall/ CRC: London, 1996.

18. Tukey JW. The Problem of Multiple Comparisons. Princeton University: USA, 1953.

19. van't Veer LJ, Bernards R. Enabling personalized cancer medicine through analysis of gene-expression patterns. Nature 2008;452: 564-570.

20. Glas A, Floore A, Delahaye $\mathrm{L}$, et al. Converting a breast cance microarray signature into a high-throughput diagnostic test. BMC Genomics 2006;7:278

21. Aslam Ml, Taylor K, Pringle JH, et al. MicroRNAs are novel biomarkers of colorectal cancer. Br J Surg 2009;96:702-710.

22. Negrini M, Nicoloso MS, Calin GA. MicroRNAs and cancer-new paradigms in molecular oncology. Curr Opin Cell Biol 2009;21: 470-479.

23. Wang QZ, Xu W, Habib N, et al. Potential uses of microRNA in lung cancer diagnosis, prognosis, and therapy. Curr Cancer Drug Targets 2009;9:572-594.

24. Paranjape T, Slack FJ, Weidhaas JB. MicroRNAs: tools for cancer diagnostics. Gut 2009;58:1546-1554.

25. Garzon R, Calin GA, Croce CM. MicroRNAs in cancer. Ann Rev Med 2009;60:167-179.

26. Li J, Smyth P, Flavin R, et al. Comparison of miRNA expression patterns using total RNA extracted from matched samples of formalin-fixed paraffin-embedded (FFPE) cells and snap frozen cells. BMC Biotechnol 2007;7:36.

27. $\mathrm{Xi} Y$, Nakajima $G$, Gavin $E$, et al. Systematic analysis of microRNA expression of RNA extracted from fresh frozen and formalin-fixed paraffin-embedded samples. RNA 2007;13:1668-1674.

28. Hui $A B Y$, Shi W, Boutros PC, et al. Robust global micro-RNA profiling with formalin-fixed paraffin-embedded breast cancer tissues. Lab Invest 2009;89:597-606.

29. Glud $M$, Klausen $M$, Gniadecki $R$, et al. MicroRNA expression in melanocytic nevi: the usefulness of formalin-fixed, paraffin-embedded material for miRNA microarray profiling. J Invest Dermatol 2009; 129:1219-1224.

30. Ma Z, Lui W-O, Fire A, et al. Profiling and discovery of novel miRNAs from formalin-fixed, paraffin-embedded melanoma and nodal specimens. J Mol Diagn 2009;11:420-429.

31. Hlubek F, Brabletz T, Budczies J, et al. Heterogeneous expression of Wnt/beta-catenin target genes within colorectal cancer. Int J Cancer 2007:121:1941-1948. 